\title{
Tracce floriane negli archivi svizzeri: documenti concernenti gli umanisti Michelangelo e John Florio conservati a Coira, Berna e Zurigo ${ }^{\mathrm{I}}$
}

\author{
Carla Rossi \\ Universität Zürich
}

\begin{abstract}
Il contributo offerto dal lessicografo e traduttore John Florio e da suo padre Michelangelo alla cultura italiana in esilio nell'Inghilterra rinascimentale è stato particolarmente rilevante. Dopo un lungo peregrinare che lo portò dalle carceri di Tor di Nona sino a Londra, dove si trattenne per un tempo assai breve, parlando solo italiano e latino, poi a Strasburgo ed infine sui monti della Bregaglia, Michelangelo Florio si spense sicuramente a Soglio nel I566, insieme alla propria moglie, lasciando tre figli in tenera età. $\mathrm{Da}$ «Soi», John partì, ancora adolescente, nuovamente alla volta dell'Inghilterra. Le indagini archivistiche condotte in primo luogo in Svizzera, ma anche in Italia, Francia, Germania e Inghilterra, hanno permesso di seguire le tracce dei due Florio, di cui si fornirà qui una breve panoramica.
\end{abstract}

Keywords: Michelangelo Florio, John Florio, William Shakespeare.

Nel 2016, in occasione dei quattrocento anni dalla scomparsa di William Shakespeare sono tornate in auge ipotesi e teorie fantasiose, senza alcun fondamento scientifico, sulla presunta italianità del Bardo, germogliate in Italia durante il Ventennio fascista. Michelangelo e suo figlio Giovanni Florio sono stati proposti nuovamente quali veri autori del ricco corpus shakespeariano, mentre attorno alle loro figure è sorta una vera e propria industria, fondata su ricostruzioni biografiche fittizie attraverso siti web, sedicenti associazioni culturali, libri editi con il metodo dell'auto-pubblicazione (dunque senza il controllo di alcun comitato scientifico) e presunte librerie specializzate. Risale al giugno del 2017 la notizia del sensazionale ritrovamento dell'atto di nascita di Michelangelo Florio, alias William Shakespeare, che così recita: «Ufficiale: Shakespeare nacque a Messina»²,

I Il presente contributo riprende parzialmente lo studio su Florio in Rossi 2018: 95-I30, volume pubblicato grazie ad un finanziamento del Fondo Nazionale Svizzero per la Ricerca Scientifica.

2 Così recita la notizia fasulla, data dal sito sky24ore.it (il cui nome è appositamente giocato sulla similitudine con la nota testata giornalistica televisiva; chiuso nel 2017): «Quella che fino ad oggi si riteneva essere solo una leggenda si è rivelata realtà: William Shakespeare era, in realtà, originario di Messina. La conferma arriva dal Centro Studi Shakespeariano di Stratford-upon-Avon, fino a ieri considerata la città natale di Shakespeare. Il 26 giugno 2017 è stato, infatti, recuperato l'atto di nascita di Shakespeare, fino ad oggi mancante. Il documento è stato rinvenuto nell'archivio Shakespeare della biblioteca di Stratford-upon-Avon, in mez- 
purtroppo subito riverberata in rete da giornalisti poco avvertiti, non al corrente del fatto che gli atti di nascita vennero istituiti, in Italia, solo dal 28 giugno I8I5. Per ricostruire le date di nascita dei personaggi venuti al mondo prima del I8I5, si fa ricorso esclusivamente alle trascrizioni dei nomi nei registri battesimali, obbligatori per la Chiesa Cattolica dopo il Concilio di Trento (I563), ma già in uso dal Medioevo. La confusione e la conseguente possibilità di dare libero sfogo alla fantasia di chi ha voluto plasmare personaggi fittizi per rendere verosimile l'idea dell'italianità del Bardo derivano in primo luogo dalla pessima abitudine degli scriventi di ripetere informazioni obsolete senza condurre puntuali (e impegnative) ricerche d'archivio. Per questo motivo, i dati anagrafici relativi a Michelangelo Florio paiono, ad un primo esame, piuttosto labili e quindi facilmente manipolabili.

In realtà, è possibile ricostruire un quadro esaustivo dell'esistenza di questo singolare, inquieto e proteiforme umanista, nato a Firenze da genitori ebrei convertiti, prima francescano, poi apertamente lutherano (Rossi 20I7: IO4-I2O).

Ricercato dall'Inquisizione nel maggio del I550, incarcerato a Roma a Tor di Nona e poi rocambolescamente evaso, Michelangelo Florio fu costretto ad un periplo della penisola italiana, per riparare a Londra, dove fu pastore, per un anno (I550-I55I), della piccola comunità italiana. Qui, si macchiò del crimine di violenza carnale (ai danni della propria perpetua), come risulta dalle carte superstiti; perdonato dal Vescovo della città, ma sospeso dall'incarico e costretto a sposare la vittima, rimasta incinta, fu cacciato dal paese con tutti gli stranieri protestanti all'avvento di Maria la Cattolica. Fuggì a Strasburgo, con la famiglia, giungendo infine nel Canton Grigioni, a Soglio.

La ricostruzione della biografia di Michelangelo Florio e di suo figlio Giovanni, destinato a divenire uno dei maggiori lessicografi europei del Cinquecento, è stata possibile grazie a puntuali ricerche d'archivio, condotte anche in Svizzera, in particolar modo analizzando le carte bregagliotte confluite sia presso l'Archivio di Stato dei Grigioni, sia presso la Burgerbibliothek di Berna (penso in particolare al ms. A. 93. 7. II, che conserva documenti relativi al Sinodo del I57I, importanti per la ricostruzione della data di morte di Michelangelo), ma anche studiando il prezioso materiale relativo a Scipione Lentolo (conservato presso l'Archivio di Stato di Zurigo) ${ }^{3}$, e la sua diretta testimonianza in merito a Michelangelo Florio, oltre, naturalmente, alla corrispondenza di Heinrich Bullinger ${ }^{4}$.

zo ai documenti personali dello scrittore. Dall'atto di nascita si evince che Shakespeare si chiamava in realtà Michelangelo Florio ed è nato a Messina il 23 aprile I564. Lo scrittore era in realtà figlio di Giovanni Florio, un medico calvinista, e di una nobildonna, Guglielmina Scrollalanza».

3 Zürich, Staatsarchiv, E II 380.

4 Dal 2009 mi occupo tanto della ricostruzione biografica di Michelangelo Florio e di suo 
Nel contesto della cosiddetta questione shakespeariana va notato che i sostenitori della tesi che vorrebbe i due Florio (Michelangelo come "mente" e John come "braccio") attivi nel forgiare a Londra, dai primi anni Novanta del Cinquecento, il fantoccio di un commediografo dal nome anglosassone dietro cui nascondersi, non hanno mai tenuto in debita considerazione le date. Ad esempio, se, tra le carte conservate nel Regno Unito, non vi è traccia alcuna del rientro a Londra di Michelangelo, la ragione di tale assenza non è certo da imputare alla disattenzione dei responsabili dei vari censimenti degli stranieri (Kirk-Kirk I90o-I9IO), come vorrebbero alcuni, quanto piuttosto ad un evento (tra l'altro, piuttosto prevedibile) occorso nel I566 e di cui abbiamo testimonianza certa grazie ad un documento conservato presso l'Archivio di Stato di Coira: la morte, a Soglio, di Michelangelo durante la tremenda epidemia di peste nera che colpì l'Europa nel I566.

Come accennato, Michelangelo ebbe una vita burrascosa e il suo percorso di fede non fu affatto lineare: fondamentalmente inviso ai più per le sue idee eterodosse, per la foga dei suoi concioni e per la sua condotta morale, visse costantemente in fuga in varie città europee, sino a giungere nei Grigioni, in quella frazione italofona, appartenente alla comunità delle Tre Leghe Grigie, che all'inizio del secolo avevano abbracciato il Protestantesimo, terra d'esilio di molti italiani considerati eretici a causa dell'adesione alla nuova dottrina riformata. Sui frontespizi delle sue opere egli appare spesso come «Florentino già predicatore famoso del Sant'Evangelo in più città d'Italia et in Londra» ${ }^{5}$. Sul registro dei partecipanti al sinodo di Coira del I56I troviamo la sua firma al numero i9 quale: «Michaël Angelus Florius

figlio Giovanni, quanto dell'edizione dell'opera omnia di entrambi. Oltre ad alcuni articoli per la rivista Theory and Criticism of Literature and Arts, segnalo la pubblicazione a Londra di un primo volume di studi floriani, dedicato a Giovanni Florio (più noto in Inghilterra col nome anglicizzato di John): cfr. Rossi 20I8. In questo contesto va segnalato che da alcuni anni l'Institut für Schweizerische Reformationsgeschichte dell'Università di Zurigo ha intrapreso il progetto di edizione digitale delle circa dodicimila lettere ricevute da Bullinger (Bullinger-Briefwechseledition, http://teoirgsed.uzh.ch).

5 Opere che, oltre alla già citata Apologia, datata Soglio 4 settembre $\mathrm{I} 556$, sono: $\mathrm{I}^{\circ}$ Regole de la lingua thoscana e Regole et instituzioni de la lingua thoscana: due redazioni manoscritte diverse di un manuale di italiano redatte durante il soggiorno inglese; i testi sono stati pubblicati in Pellegrini 1954, n. I4. La versione della grammatica dedicata a Henry Herbert conte di Pembroke, nel 1553 fresco cognato di Jane Gray per averne sposato la sorella in maggio, è conservata manoscritta sotto la segnatura Dd. XI. 46 presso la Cambridge University Library, datata Londra 2I agosto I553. Un'altra copia autografa e quasi identica, ma priva di data e dedicata a Jane Gray, è conservata nel manoscritto Sloane 30II della British Library. $2^{\circ}$ Catechismo cioè forma breve per amaestrare i fanciulli, S. Mierdman, London 1553: traduzione italiana del catechismo di John Ponet, vescovo di Winchester (ad uso della comunità degli esuli italiani in Inghilterra). $3^{\circ}$ Historia de la vita e de la morte de l'illustrissima signora Giovanna Graia già regina eletta e publicata d'Inghilterra, R. Schilders, I607: biografia di lady Jane Grey e, al contempo, libello contro Maria Tudor, che Florio non pubblicò in vita ed uscì postumo. 
Flor[ent]inus, Sol[iens]is minister ${ }^{6}$, nella stessa forma in cui compare nei suoi protocolli notarili superstiti (I564-I566).

In un estratto notarile del 29 giugno I573, presente nel faldone dei notai Giovanni e Andrea Ruinelli ${ }^{7}$ ci si riferisce a lui come «Michelangelo Florio Fiorentino, defunto, e già ministro della chiesa di Soglio» ${ }^{8}$.Questo documento (fig. 2) è stato erroneamente considerato un testamento da chi si è occupato della ricostruzione biografica di Michelangelo, eppure è evidente che non si tratta affatto di un lascito testamentario, ossia di un atto giuridico unilaterale non recettizio mortis causa firmato da Florio, mediante il quale egli dispone dei propri beni. Nel faldone notarile dei Ruinelli, tra l'altro, ogni testamento è indicizzato come tale (testamentum).

L'atto in questione è indicizzato come «Designatio Constantiæ filiæ D[omini] Michaelis Angeli, p[er] ad[vocat]os relic[torum] filio[rum] 29. Junij», cioè «Designazione di Costanza, figlia del Signor Michelangelo, da parte dei rappresentanti legali dei figli superstiti» (fig. I) ${ }^{9}$.

Nonostante gli sforzi profusi da Corrado Panzieri, nel tentativo di provare che Michelangelo Florio fosse ancora vivo e attivo dopo il 573 (Panzieri 20I4), risulta palese che la data di lunedì 29 giugno I573 vada presa come terminus a quo per considerare Florio ormai defunto ${ }^{\mathrm{IO}}$.

Ecco la trascrizione dell'estratto notarile:

Pro Constantia extractum est ${ }^{\mathrm{II}}$.

AND [Anno Nativitatis Domini] I573. Die lunæ 29 Junij.

6 La firma autografa di Michel Angelo Florio è al numero I9 di pagina 98 del libro degli iscritti al Sinodo di Coira e non al numero 20 dell'elenco, come riportato dal pastore Truog (che commise vari errori nelle proprie annotazioni), in Truog I90I: 6 (Teil I, I555-I76I).

7 Italianizzo qui di seguito i nomi dei due notai Ruinelli, padre e figlio, che figurano in latino come Johannes e Andreas Ruinelli (Ruinella, de Ruinello, de Ruinellis); si veda ad es.: «Ego Johannes filius Andreae molitoris de Ruinellis», nei Protocolli notarili di Soglio, faldone XV, folio $2 v$. Sul padre, Johannes, discendente da una famiglia di mugnai e sposato con Anna von Salis, cfr. Pool 1983: 74; 76-77 e, sulla famiglia Ruinelli, Pool 1983: I07 sgg.

8 Il testo è conservato presso lo Staatsarchiv Graubünden di Coira, sotto la segnatura B $663 / 27$, c. 48r/v (B 663/I-44: Bergeller Notariatsprotokolle, B 663/27: Protokoll geführt von Johannes Ruinello und Sohn Andreas, Jahr 1573). Nella presente trascrizione correggo le numerose sviste, anche di accordo di casi, presenti nella copia fornita da Gianna Martinoli nella sua comunque pregevole tesi di laurea depositata presso l'Università degli Studi di Milano (Martinoli 1997/I998). Integro le parole non trascritte, sciolgo doverosamente le abbreviazioni e correggo, inoltre, il manoscritto laddove figura rassis, lapsus calami per cassis, per influenza della $r$ di blancaria. Il manoscritto dei protocolli notarili di Florio è conservato sempre presso l'Archivio di Stato di Coira, cfr. Hoiningen-Huene (I9I7: 97-IO5, 2OI-2II, 388-396; I9I9: 57-6I, 85-95, I54-I60, I87-I89).

9 Chur, Staatsarchiv Graubünden, cod. B 663/27, c.Iov.

Io Johannes Ruinella e i suoi figli furono notai a Soglio, con mandato per tutta la valle Bregaglia, dalla metà sino alla fine del XVI secolo. Il figlio di Johannes, Andrea, fu in seguito rettore della scuola di latino istituita a Coira ( 1578 bis I6I6).

II La grafia è differente da quella della mano che ha trascritto l'atto. 


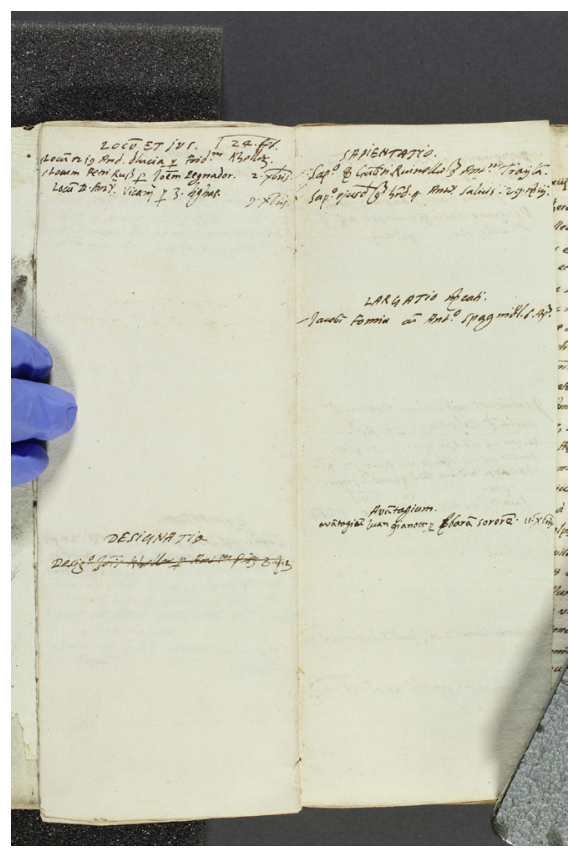

Fig. I: Chur, Staatsarchiv Graubünden, cod. B 663/27, c. Iov.

Magnificus Dominus Antonius à Salicibus, alias dignissimus Valtæ [per Vallistellinæ=Valtellina] Vicarius, discretus ${ }^{12}$ ser Johannes de Raphaël Curtæbat[is], ac cum eis ser Andreas, filius quondam Augustini Duttæ, tamquam advocati legitimi hæredum quondam Domini Michaëlis Angeli Florji florentini, alias fidelis verbi Dei Soliensis ecclesiæ ministri ${ }^{13}$, dederunt ac designarunt Dominæ Constantiæ filiæ quondam dicti Domini Michaëlis omnia mobilia, sive blancaria, cum rassis ${ }^{14}$ duabus, quæ fuerunt quondam

I2 Va segnalato, dopo aver esaminato gli altri atti stilati dai due Ruinelli, che è costume dei due notai, impreziosire i nomi dei dignitari della valle con attributi quali honestus, probus, discretus, che tornano spesso nelle loro carte.

I3 Si noti l'uso dell'espressione latina «fidelis verbi Dei Minister», che ricorre in moltissimi documenti coevi a indicare la funzione di pastore protestante. Cfr. ad esempio, per le occorrenze, Strohm (1996: 510).

I4 Trovo interessante, dal punto di vista linguistico, in questo atto, la presenza del termine rassa a indicare la sottana. In Du Cange I883-1887 la voce viene registrata come «Vestis genus, tunica rudis, aspera, non mollis». Anche in Bierbach (1997:340) il termine dialettale viene segnalato come ancora in uso nel Nord Italia e in Bregaglia, proprio a Soglio: «Berg. rassa f. 'gonnella'; bregagliotto (Soglio) 'sottana'». Dunque, non solo il contenuto dell'atto, ma anche l'uso di questo particolare termine, attestato localmente, sono chiari indizi che a redigerlo non è stato certamente Michelangelo Florio (come sostengono sia Panzieri 20I4: 42 sia Romani-Bellini 2012: 192). Florio, da umanista toscano, non avrebbe mai utilizzato un termine dialettale bregagliotto. Il fatto che Michelangelo, in veste di notaio, non utilizzasse e a volte addirittura 


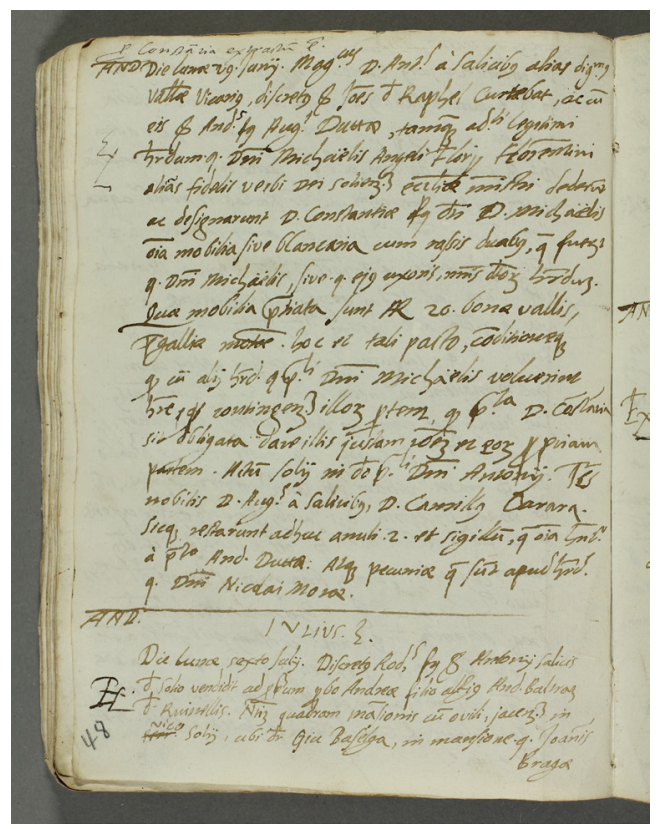

Fig. 2: Chur, Staatsarchiv Graubünden, cod. B 663/27, c. 48 r/v.

Domini Michaëlis, sive quondam ejus uxoris, matris dictorum hæredum. Quæ mobilia pretiata sunt Florini 20 bona vallis Pregalliæ moneta; hoc est tali pacto, conditioneque quod cum alij hæredes quondam predicti Domini Michaëlis voluerint habere quamvis contingentem illorum partem quod predicta Domina Constantia sit obligata dare illis justam rationem et eorum propriam partem.

Actum Solij, in domo predicti Domini Antonij.

Testes: Nobilis Dominus Augustus à Salicibus, Dominus Camillus Carara.

non comprendesse l'uso di termini locali è attestato dall'utilizzo che egli fa del termine hypocaustum, ipocausto. Infatti, in tutti gli atti rogati a Soglio nella cosiddetta stüa (l'unico locale riscaldato della casa alpina attraverso un sistema di ipocausto, appunto) i notai erano soliti indicare «in meo hypocausto». Ma Florio sbaglia e scrive sempre «in meo hippocausto», con un ipercorrettismo tipico di chi aveva maggiore dimestichezza con il greco, facendo derivare così il termine erroneamente da 'cavallo' híppos). In italiano antico la rascia era il tessuto nero usato nelle chiese in occasione dei funerali. John nel suo dizionario, alla voce Rascia, nel 1598 indica che si tratta di «a kinde of stuffe called silke rash»; nel I6II nota semplicemente: «Rascia, the stuffe called Rash», cfr. A Worlde of Wordes, Or Most copious and exact Dictionarie in Italian and English, collected by Iohn Florio, printed at London, by Arnold Hatfield for Edw. Blount, 1598 e Queen Anna's New World of Words or Dictionarie of the Italian and English tongues, Collected, and newly much augmented by Iohn Florio, Reader of the Italian vnto the Soveraigne Maiestie of Anna, Crowned Queene of England, Scotland, France and Ireland, Ec. And one of the Gentlemen of hir Royall Priuie Chamber. Whereunto are added certaine necessarie rules and short observations for the Italian tongue, in London, I6II, for Edward Blount and William Barret. 
Sicque restarunt adhuc anuli 2 et sigillum, quæ omnia habentur à predicto Andrea Dutta. Atque pecuniæ quæ sunt apud hæredes quondam Domini Nicolai Moræ.

[Traduzione:

«A favore di Costanza viene estratto il presente atto.

Nell'anno della natività del Signore I573. Il giorno lunedì 29 giugno.

Il Magnifico Signor Anton von Salis, noto anche come virtuosissimo vicario della Valtellina, il saggio Messer Giovanni di Raffaele de Curtebatis e con lui Messer Andrea, figlio del fu Agostino Dutta, in veste di legittimi rappresentanti degli eredi del defunto Signor Michele Angelo Fiorentino, noto anche come pastore della chiesa di Soglio, hanno assegnato in rappresentanza dei suddetti eredi, alla Signora Costanza, figlia del defunto Signor Michele, tutta la mobilia così come la biancheria con due sottane, che furono del fu Signor Michele o della sua defunta moglie, madre dei detti eredi. Mobili che sono stati stimati 20 fiorini di moneta buona della valle Bregaglia ${ }^{15}$. Questo a patto e a condizione che, ove gli altri eredi del suddetto Signor Michele pretendessero una qualsivoglia porzione della loro parte, la signora Costanza sia obbligata a dar loro la giusta quota e la parte loro spettante.

Redatto a Soglio, a casa del suddetto Signor Anton [von Salis].

Testimoni: Il nobile Signor August von Salis e il Signor Camillo Carara. E così sono avanzati due anelli e un sigillo. Tutti gli oggetti si possono avere dal predetto Andrea Dutta e il denaro è presso gli eredi del defunto Signor Nicola Mora»].

Va rilevato come la credibilità delle ricostruzioni biografiche relative al rientro in Inghilterra di Michelangelo Florio (e quindi le illazioni in merito alla sua diretta collaborazione con Shakespeare), dipendono, ovviamente, dall'anno della sua morte: l'attore e drammaturgo nacque infatti a Stratford nel I564 e l'epoca di composizione della maggior parte dei suoi lavori si ascrive ai circa venticinque anni compresi tra il I588 e il I6I3 (anno in cui Michelangelo Florio, se realmente nato nel I5I8, avrebbe avuto la veneranda età di 95 anni).

Nel rogito, si nota l'uso ripetuto dell'avverbio «quondam», rimasto ancor oggi nel linguaggio curiale e giudiziario per indicare che la persona di cui si sta parlando è defunta. Dal Medioevo in poi, l'avverbio è usato negli atti notarili davanti al nome di una persona scomparsa, a designare, appunto, «il fu».

I5 «Bona moneta» è un'espressione idiomatica che compare in moltissimi rogiti per designare purezza e peso delle monete, cfr. Zagni ( $1988, n$. 30). Il ricorso a questa espressione negli atti notarili appare una consuetudine da far risalire già alla prima metà del xII secolo. Essa dunque non va interpretata come prova dell'esistenza di una moneta migliore di una precedente in circolazione, quanto piuttosto come dettaglio meramente scritturale, relativo all'uso di una moneta "di buona qualità" - di giusto peso, non calante - per la liquidazione dell'importo pattuito nel contratto. 
Che non ci siano dubbi in merito all'uso nel presente documento è testimoniato anche dall'occorrenza dell'espressione «Andreas filius quondam Augustini Duttæ».

Per precisare, inoltre, il grado di parentela tra Costanza e Michelangelo è chiaramente indicato che si tratta della «filia quondam Domini Michaëlis», ossia della «figlia del fu Signor Michele».

Non si deduce da quale sezione di questo atto, che pure cita, Panzieri ricavi la notizia che Costanza fosse figlia di una governante di Florio, «una povera donna del posto rimasta sola, forse vedova o ragazza madre con una figlia di nome Costantia, alla quale egli lascerà quant'altro rimaneva di valore in casa. Michelangelo Florio lasciò Soglio in incognito presumibilmente nell'autunno del 1577 diretto a Oxford dove aveva preso residenza il figlio John» (Panzieri 20I4: 44).

$\mathrm{Al}$ di là del fatto che, come ho dimostrato nel già citato volume su John Florio, nel 1577 il linguista non si trovava sicuramente ad Oxford, è abbastanza inverosimile che il «quondam» Michelangelo potesse lasciare Soglio, se non "in spirto".

A Costanza, dalle mie ricostruzioni unica figlia di Florio rimasta in Bregaglia (tanto Michelangelo, quanto sua moglie, sulla base di questo documento, risultano già defunti nel 1573), spettano, per decisione dei tre rappresentanti legali dagli altri eredi di Michelangelo (che, evidentemente, non si trovavano a Soglio nel I573), mobili e biancheria, giacché la ragazza aveva evidentemente raggiunto la maggiore età nel 1573 , o stava per sposarsi ${ }^{16}$.

Bisogna evidenziare, nel documento, alcune spie interessanti: la mobilia è stata venduta prima della stesura dell'atto, come esplicitato dall'indicazione fornita in calce, per 20 fiorini della valle Bregaglia e il denaro si trova presso gli eredi del notaio Nicola Mora (di Piuro), di cui è possibile ricostruire la data di morte: maggio del $157 \mathrm{I}^{\mathrm{17}}$. Piuro era il centro pulsante dell'eterodossia degli esuli italiani sociniani anabattisti e antitrinitaristi, abitata per un decennio (1560-157I) da personaggi estremamente vicini a Florio, quali

I6 Sono queste le uniche condizioni, in tutta Europa, alle quali sarebbe potuta entrare in possesso dell'eredità. In merito al raggiungimento della maggiore età, ossia I8 anni per gli uomini, che in valle Bregaglia concedeva i diritti civili, come quello di voto per alzata di mano e, ad esempio, di esercizio della professione di notaio, e I6 per le donne si veda Pool I983: 78; Crivelli 1978: 872: «Grossjährig wurde man mit achtzehn Jahren, als man jedoch bereits Meister seines Fachs war. Das bedeutet, dass man nicht automatisch aus Altersgründen grossjährig wurde, sondern erst von achtzehn Jahren an aufwärts, wenn man eine gewisse professionelle Reife erreicht hatte»; cfr. anche Roth 2016, in particolare la sezione 4.4.3. Per le donne la maggiore età (da non confondere con l'età minima per contrarre matrimonio) significava poter siglare in autonomia contratti e altri negozi giuridicamente validi.

I7 Da un documento riprodotto nelle Landeskarten 1974: 446 risulta che il notaio morì dopo il 23 maggio del I57I (dagli archivi della famiglia von Salis, in Familienarchiv von Salis I898, risulta che Nicola Mora era attivo come notaio almeno dal I545). Dagli stessi archivi si deduce che gli eredi in questione si chiamano Johannes e Michael. 
non solo il Torriano, ma Camulio e Sadoleti, Bartolomeo Silvio, Battista Bovio, il modenese Filippo Valentino, Battista da Serravalle, e città natale di Lorenzo Lumaga, ricco personaggio locale, commerciante di stoffe. Gli "eretici italiani" vennero scacciati dai Grigioni dal Sinodo nel giugno del I57I: non è escluso che tra costoro si trovasse il padrino di uno dei figli di Florio (probabilmente proprio di Costanza) e che per questo motivo la vendita della mobilia venisse trattata da un notaio del centro presso il quale, si può avanzare l'ipotesi (dubitativa), era andata a vivere la giovane orfana, secondo gli impegni assunti dai compari al battesimo ${ }^{18}$. Il fatto che, evidentemente, Nicola Mora avesse trattato la vendita di tutta la mobilia dei Florio prima del I57I e avesse tenuto presso di sé i 20 fiorini in possesso dei quali Costanza sarebbe potuta entrare una volta divenuta maggiorenne è un'ulteriore conferma che Michelangelo doveva essere deceduto prima di quella data.

La nota iniziale «Pro Constantia extractum est» sta a significare, in gergo notarile, che il documento venne stilato su un originale, più lungo, da cui le indicazioni sono desunte e che l'estratto nulla aggiunge e nulla toglie all'originale del quale rappresenta una riproduzione parziale. All'inizio dell'atto è inoltre specificato che sono stati i maggiorenti della città (Anton von Salis, Giovanni di Raffaele de Curtebatis - il quale in altri documenti coevi figura anche come Johannes de Raphel de Kurtebat o Zuan Raphaël de Curtebatis de Solio - e Andrea, figlio del defunto Agostino - detto Bastiano - del Dotta, nonché parente di von Salis, avendo sposato una Margaretha von Salis) a decidere (evidentemente tramite un altro atto notarile, di cui il presente è, appunto, un estratto) quali beni del $f u$ Michelangelo dovessero essere ereditati da Costanza: questo perché evidentemente Florio in vita non aveva redatto testamento.

Il particolare mi pare degno di nota, in quanto indica che Michelangelo, piuttosto che fuggire altrove (come ipotizza Panzieri addirittura nel I577, ben quattro anni dopo la testimonianza di questo documento del 1573 che lo dà per defunto), doveva essere stato colto da morte improvvisa: se fosse stato lungamente malato, avrebbe certamente provveduto, da notaio qual era, a stilare le sue ultime volontà, per provvedere ai propri figli.

Va rilevato altresì come l'atto non sia stato stilato presso il notaio Ruinelli che lo sottoscrive, come era solitamente costume in Bregaglia ${ }^{19}$, ma a casa di Anton von Salis, che risulta avere qui un ruolo preminente, in veste anche

I8 Compito del padrino e della madrina di battesimo era, allora come oggi, di assistere i genitori nell'educazione religiosa del bambino e di occuparsi di sostituirsi a loro in caso di decesso.

I9 Cfr. Pool (1983: 85). Nel caso in cui l'atto sia rogato dal notaio nella propria abitazione si ha l'indicazione «aedium abitationis». Quando l'atto era rogato nella cosiddetta stüa (l'unico locale riscaldato della casa alpina) il luogo è chiaramente indicato come hypocaustum. Cfr. anche sopra, nota I4. 
di rappresentante legale degli altri eredi. Dal documento è altresì manifesto come tutti gli eredi fossero figli dello stesso letto (quindi l'ipotesi di un secondo matrimonio di Florio in Bregaglia viene a cadere): la precisazione «ejus uxoris, matris dictorum hæredum» significa proprio che la moglie di Florio, madre degli eredi inclusa Costanza, fu una sola.

Anche in un altro atto, in cui si decide di un passo alpino, rogato sempre a Soglio da Johannes Ruinelli il 27 maggio del I570, «in hypocausto Domini Vicarii Antonii Salis ${ }^{20}$, lo stesso Anton von Salis appare, come nel presente documento, quale «praeclarus Dom. Antonius, nunc Vallistellinæ Vicarius»; ma è indicativo che di questa eredità così poco rilevante si occupi addirittura il vicario della Valtellina ${ }^{21}$, il cui coinvolgimento appare giustificato dalla responsabilità della famiglia von Salis di Soglio nei confronti dei giovani eredi di un personaggio pubblico quale fu Florio, a ulteriore conferma che i suoi figli erano rimasti orfani di entrambi i genitori. Sulla base di questo atto notarile, diviene inattendibile l'indicazione fornita dal pastore Truog, secondo il quale Michelangelo Florio si trasferì in Inghilterra nel $1577^{22}$.

Purtroppo le informazioni di Truog sono frammentarie e incomplete: non solo egli sbaglia il numero di registro della firma di Florio sul libro degli iscritti al sinodo di Coira del 556 (che dà erroneamente al numero 20, invece che al 19), ma nella lista dei pastori di Soglio commette un ulteriore errore, dimenticando Giovanni Antonio Cortese da Brescia, che contribuì, con il fratello Giovan Francesco, al definitivo passaggio di Soglio alla Riforma. Truog basò la propria deduzione errata in merito alla morte di Florio sul fatto che, dai documenti da lui consultati, nel 1577 a Soglio risulta essere attivo come pastore riformato Giovanni Marzio da Siena.

All'erronea congettura non è mancata, però, fortuna ed è così che l'inesattezza si è riverberata in altri testi sulla Riforma ${ }^{23}$. Fortunatamente già Cantimori (I939: 282, 469), e più di recente Bonorand (2000: I8I) si sono prodigati per segnalare e correggere l'errore. In una svista simile, causata dalla confusione del cognome di Michelangelo con quello di Simon Florio, è caduto anche Martin Bundi, il quale ha scritto che Simone (alias Michelange-

20 Salis-Regesten, D VI A I, atto nr. 282 (Regesten zu Pergamenturkunden, I. Serie, Chur, Staatsarchiv Graubünden, segnatura generale D VI A I).

2I Vedi nota precedente. Questo Anton, in alcune carte Antonio, era figlio di quell'Augustin Salis il Vecchio, che compare in svariati atti rogati da Michelangelo a Soglio. Cfr. la Genealogia redatta da Nikolaus von Salis-Soglio (I853-I933), autografa, presso lo Staatsarchiv Graubünden, D VI So [2I/I24] II. A. 2, Quaderno IV, discendenza di Rodolfo il Lungo, Casa Augustin. Da un atto custodito presso l'archivio privato dei Salis-Soglio, oggi a Chur, Staatsarchiv Graubünden, D VI SM / Cp I87, risulta già deceduto il I6 Augusto del I604.

22 Die Pfarrer 1935: 214. Citare il libro di Truog (I865-I953) come fonte attendibile risulta estremamente azzardato, perché è evidente dalle molte approssimazioni e dagli errori commessi, che il pastore non lavorò su documenti di prima mano, verificati negli archivi.

23 Come ad esempio in Giovanoli 1932: 5I-56 e in Camenisch 1950:58. 
lo) Fiorillo (Florillo, Florio) era originario di Firenze e fu attivo come Pastore a Soglio e a Chiavenna (Gewissensfreiheit 2003: 217).

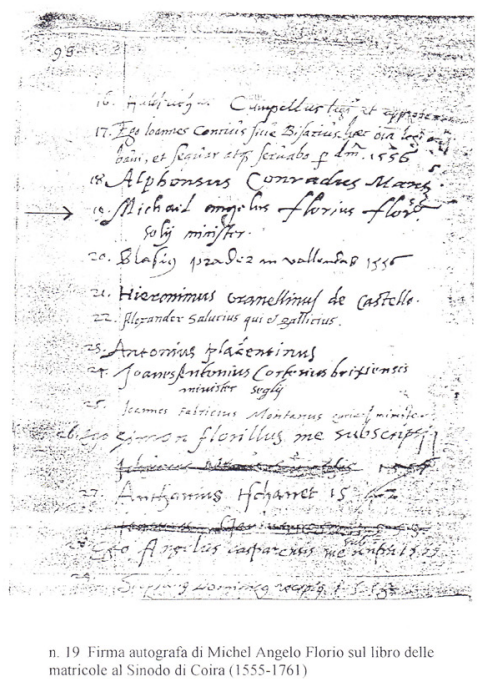

Fig. 3: Chur, Staatsarchiv Graubünden,

Libro degli iscritti al sinodo retico di Coira, anno 1556, c. 99r.

Truog pare ignorare un'ulteriore documentazione decisiva per dirimere la controversia sull'inizio dell'attività del successore di Florio. Alla fine degli anni novanta del Cinquecento, infatti, Broccardo Borrone si proponeva di riportare in seno alla chiesa cattolica quei ministri italiani considerati eretici e la sua viva testimonianza sul ministro di Soglio Giovanni Marzio da Siena, ex prete cattolico, è interessante: «apostatò una trentina d'anni fa ed ha circa 60 anni. Ha per moglie una veneziana smonacata, dalla quale ebbe due belle figliole, ora in età da marito. Scrisse un'operetta sul sacrificio della messa alla quale rispose Gio. Paolo Nazario con un'Apologia stampata nel 1597. [...] Bisogna essere furbi ed impiegare la forza per catturare questo ministro calvinista, con il quale le buone maniere si dimostrerebbero ineficaci» (Busino 1962: I48). Un'informazione assai simile si trova anche in Heilige Wiedergeburt der evangelischen Kirche in den gemeinen drei Bündten der freien hohen Rhätien oder Beschreibung ihrer Reformation und Religionsverbesse- 
rung di Bartholomaeus Anhorn (Brugg I680: 77), il quale riferisce che Johannes Martius Senensis, pastore di Soglio, era «ketzerischer Predicant» ossia pastore eretico, all'incirca dal 1567.

Nelle Memorie Domenicane del 1948 si trova la trascrizione della dichiarazione di Marzio in occasione della disputa intono all'istituzione della mes$\mathrm{sa}^{24}$. Tanto la testimonianza di Borrone, quindi, quanto quella di Anhorn lasciano dedurre che Marzio fosse pastore a Soglio da prima del I577.

Per quel che concerne la documentazione superstite relativa ai notai bregagliotti, bisogna purtroppo ricordare che sono andati perduti gli atti rogati negli anni I477-I5IO, I526-I529, I530, I53I, I534, I538, I540, I545-I548, dal I560 sino al 564 (dunque gli atti di Michelangelo in veste di notaio prima di quelli conservati nel faldone B 663/2I che s'iniziano il 20 febbraio del '64), I57I, I576-I580, I588, I589, I592, I593.

Il documento a favore di Costanza Florio che ho testé prodotto non è il solo a inficiare le teorie che vogliono Michelangelo attivo, dopo il I577, in Inghilterra al fianco del figlio John nella redazione dei testi teatrali e dei sonetti firmati da William Shakespeare. Scipione Lentolo, pastore di Chiavenna, nei suoi Commentarii del I57I parla di Michelangelo Florio come defunto già da qualche tempo ${ }^{25}$. Il I57I (data di morte anche del notaio di Piuro che ha trattato la vendita di tutta la mobilia dei Florio) costituisce senza ombra di dubbio il terminus ante quem per il decesso di Michelangelo.

È soprattutto la testimonianza di Mino Celsi durante il processo di eresia a Torriano e Sozzini, tràdita dallo stesso manoscritto della Burgerbibliothek di Berna A. 93. II, Fragmentum libri contra haereticos Hieronyimum Turrianum et Camillum Sozzinum, alle carte I $v$ - $2 r$, che non lascia dubbi sul fatto che Michelangelo fosse morto, appunto, nel 5566 (data, come già accennato, alla quale si interrompono tutti gli atti notarili da lui rogati a Soglio). Celsi, infatti, riferendosi a Michelangelo, afferma che sono trascorsi «tant'anni doppo la sua morte». Prima di analizzare il documento, ricordo brevemente i fatti:

24 Tra l'8 e il ıo marzo 1597, in un clima di accesa contrapposizione, avvenne una pubblica disputa tra cattolici e calvinisti sul tema dell'eucarestia confessionale, a Piuro. La disputa non ebbe alcun risultato concreto se non la pubblicazione di due volumetti: uno di Giovanni Marzio, forse edito a Poschiavo e l'altro del domenicano Giovanni Paolo Nazari, l'Apologia di frate Giovanni Paolo Nazari da Cremona (Como 1597), poi tradotta in latino e ampliata per essere inserita nel primo volume degli Opuscula varia theologica (Bononiae I63I, pp. 76-I54; il secondo volume uscì a Bologna nel I632). Durante la disputa Marzio si impegnò così: «Io Giovanni Marzio, ministro della comunità di Soglio, avendo già richiesto al magnifico Pretore di Piuro e agli altri Signori la facoltà di disputare col suddetto rev. parroco, prometto che non appena mi sarà concessa la facoltà richiesta, accetterò la discussione sia a voce che per iscritto, secondo piacerà ai suddetti Signori: nella discussione dimostrerò che Gesù Cristo non ha mai istituito il sacrificio della Messa e che nella S. Scrittura non si trova nemmeno una sillaba su questo argomento» (Memorie Domenicane I948: I65-166).

25 Commentarii conventus synodalis conv. mense junii I57I in oppido Clavenna, manoscritto conservato a Berna, Burgerbibliothek, Bong. cod. A. 93. 7, cc. $5 \mathrm{I} r-56 r$. 
Camillo Sozzini apparteneva a una famiglia senese accusata di eresia; sfuggì alla cattura che coinvolse i fratelli Cornelio e Dario nel I560, riparando in Svizzera. A Zurigo fu ospite del mercante Antonio Mario Besozzi: scoperto nel 1565 , fu cacciato dalla città e il Besozzi fu processato. Camillo si recò allora in Valtellina, cercando di stabilire la propria residenza a Chiavenna, ma ne fu impedito dal pastore riformato ortodosso Scipione Lentolo. Scelse di abitare a Piuro, in casa di Girolamo Torriano, già molto vicino a Florio, dove conobbe e divenne amico del mercante genovese anabattista Niccolò Camulio, «mercator nummatissimus» ${ }^{26}$. Sebbene, come abbiamo avuto modo di vedere, Florio e Torriano, nel I56I, avessero accettato la censura del Sinodo, essi rimanevano, in realtà, fermi nelle proprie convinzioni. La vicinanza di Florio al movimento spiritualista è palese anche dalle vicende che coinvolsero amici di Bernardino Ochino che, dopo l'espulsione di Ochino da Zurigo, nel I563, tentarono di capire, con una lettera a Torriano e a Florio datata 3 dicembre, se i due fossero disposti ad accogliere l'esule in Bregaglia ${ }^{27}$. La lettera indirizzata a Florio fu sequestrata e quindi egli non la ricevette mai. Ricevette, invece, una lettera dal Camulio il 20 settembre del I565, con la quale il mercante genovese gli annunciava un viaggio, imminente, di Camillo Sozzini a Piuro: «venit ut videat vestram ecclesiam, et si reperiet in ea recte habitari posse, consistet; id quod causa erit ut eo pertrahat dominem Faustum et fortassis alios» ${ }^{28}$.

Nel 1570 le autorità politiche dei Grigioni, su richiesta dei pastori riformati, in primis di Scipione Lentolo, che appena giunto a Chiavenna si era schierato contro gli eterodossi italiani, decretarono che, pena l'espulsione, gli esuli italiani dovessero professare o la fede cattolica o quella riformata svizzera, la Confessio Helvetica Posterior (pubblicata a Zurigo nel I566), accettata dal Sinodo delle chiese retiche. Ciò sembrò ad alcuni italiani una violazione della tolleranza religiosa praticata nei Grigioni e vi si opposero.

Come già anticipato, centro vivo dell'eterodossia era Piuro, dove risiedeva il maggior numero di sociniani. Il I3 giugno del I57I il Sinodo retico deliberò che «Girolamo Torriani, ministro di Piuro, sia espulso dal ministero fino al prossimo sinodo. Camillo Sozzini e Nicolò Camogli siano scomu-

26 Come è detto nel già citato codice bernese A. 93. 7 .

27 «Il povero vecchio è qui presso di noi, con quattro figliuolini, [Ochino era già vedovo] abbandonato da tutti fuorché Dio. Ho fatto quanto in me per aiutarlo. Ho insistito che i suoi figli rimanessero con me; e li terrò a casa mia finche Ochino possa comodamente sistemarsi costà. Se ciò non è fattibile, tratterrò i ragazzi presso di me. Sarà costretto a partire per l'Inghilterra, poveretto, o per la Boemia, dove sarebbe imprudente condurre i suoi figliuoli. Spero con tutto il cuore che possa venirsene ad abitare fra voi, specie dacché ha promesso di non predicare più e di ritirarsi a vita privata». Camulio accenna anche alla possibilità che Ochino si rechi ad Anversa «ove chicchesia può viver non molestato dai vecchi e dai nuovi carnefici». Cfr. Bainton (I940: I44).

28 Inclusa nel già citato codice bernese A. 93.7 , c. $49 v$. 
nicati fino al prossimo sinodo». Torriano fu rimosso dal magistero a causa dell'affermazione secondo cui «non conviene ai cristiani l'uccider gli uomini per ragion di fede [...] e [a] quei ministri che senza far le debite, christiane ammonizioni accusano gli eretici al Magistrato $»^{29}$. Il ministro di Piuro fu, in realtà, sospeso sino al 1572 quando, fatta pubblica abiura, venne riammesso. Camulio, imparentato nei Grigioni con i von Salis e i von Planta, con il suo «aetario locupletissimo» (il suo capitale era valutato 30.000 fiorini d'oro) era reo di aver finanziato l'«asylum hæreticis profugis», ossia il soggiorno di molti italiani compromessi dottrinalmente: Antonio da Padova, Francesco Vacca, Filippo Valentini, Pietro Romano, Lodovico Fieri, Battista Bovio. Un vasto giro di persone unite nella comune tendenza al radicalismo religioso e sociale. Tutto questo gruppo, compreso Camillo, venne espulso nel I57I.

Durante il Sinodo del I57I, il senese Mino Celsi difese il principio della tolleranza, affermando che l'autorità civile non aveva il diritto di punire gli eretici "purché in tutto ciò che non è stretta materia dottrinale osservino le leggi civili e morali». Celsi tentò soprattutto di tutelare Torriano dalle accuse di aver accolto nella sua chiesa eretici scomunicati e di aver frequentato Michelangelo Florio, in questi termini:

Dicono gli accusatori ch'egli ha conversato con heretici, et in particolare con messer Michel'Angelo già Ministro di Soj. Oh Dio che sento io! Uno che, vivendo, non è stato mai, non in pubblico appresso la Sinodo, né in particolare accusato o imputato o pur posto in sospetto di mala dottrina, hoggi morto, è nominato in una sinodo christiana per heretico e punito chi ha conversato seco [...]. Anzi, di più: è presente chi ha una confession di sua mano [di Florio stesso, NdA], pia e christiana, e non lo difende da tanta calunnia. Ma poniamo anco ch'egli fosse stato heretico, debbesi, tant'anni doppo la sua morte, punire un ministro e danneggiare una chiesa per aver egli conversato con esso lui se non si prova che questo tale abbia bramato, approvato e predicato la sua falsa dottrina!

Molti italiani lasciarono per sempre i Grigioni, altri, come Torriano, si dissero pronti all'abiura. Nel I572, per riesaminare i singoli casi venne creata una commissione ${ }^{30}$, che si mise in viaggio verso Piuro, fermandosi prima a Soglio, in casa di Giovanni Antonio Cortese, per perquisire la sua casa e vedere se nella sua biblioteca conservasse libri dell'Ochino (Zucchini I990: 542).

A conferma della morte di Michelangelo a Soglio, si legga anche quanto scritto da Pietro Domenico Rosio de Porta, il quale, grazie ai suoi contatti con i von Salis e grazie all'accesso alla loro biblioteca, pare essere ben in-

29 Bern, Burgerbibliothek, Bong. cod., A. 93. 7. II, c.Ir.

30 Sull'intera questione cfr. il ricco contributo di Zucchini 1990. 


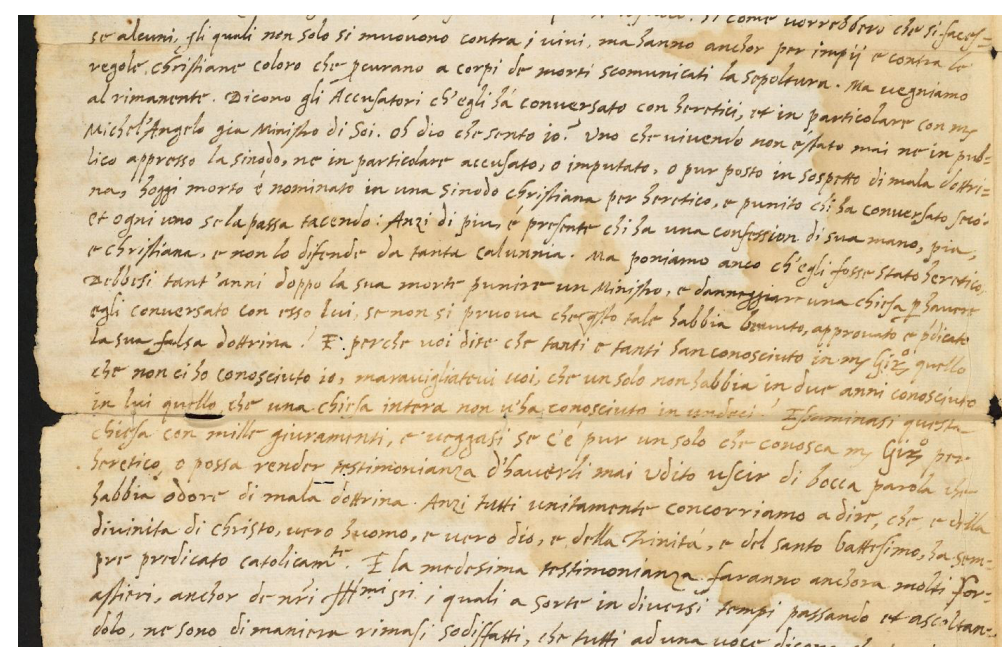

Fig. 4: Bern, Burgerbibliothek, Bong. cod. A. 93. 7. II, c. Ir.

formato riguardo agli eventi di Soglio, nella sezione della sua storia delle chiese retiche riformate in cui passa in rassegna i pastori di Soglio:

SOLIO [...] Primo Lactantius Bergomensis [...] ei succedit Michael Angelus Florius per complures annos, vir doctus et facundus, Camilli placitis addictor, pacis interea studiosus, ibidem defunctu (Rosius a Porta I776: 48).

Va sottolineato, a scanso di equivoci, che l'indicazione «ibidem defunctus» indica, appunto, che Michelangelo morì a Soglio.

A tutto ciò si aggiunga, quale ulteriore tassello del composito mosaico che si va faticosamente ricostruendo, che l'archivio privato dei von Salis di Soglio ${ }^{31}$ ha conservato per secoli un documento, oggi presso l'Archivio di Stato dei Grigioni (sotto la segnatura D VI SM / R 82), che porta la data del primo aprile del I569, quando Jacobus à Zun, «publicus Imperiali Auctoritate Notarius» attivo a Soglio, traduce dal latino in italiano un atto stilato da «Michael. Florius p[ublicus] Not[arius]» tre anni prima (di cui sono riuscita agevolmente a rinvenire l'originale latino, datato I aprile I566, ai foll. 20-2I del faldone B 663/2I degli atti rogati da Michelangelo) in merito ad una banale controversia tra gli eredi di Andrea Ruinelli e quelli del Magister Johan-

3I Privatarchiv von Salis-St. Margrethen Dauerdepositum des Familienverbandes der von Salis. In merito a questo archivio, bisogna notare come un lavoro di schedatura corretta degli atti sia ancora tutto da effettuare. Il documento D VI SM / R 82, ad esempio, è schedato sotto il nome del notaio che ha tradotto l'atto, indicando erroneamente Michelangelo Florio come «Michael Florinus Vonzun». 
nes e del Magister Rodolfo, di cui Agostino von Salis, deceduto proprio nel I563, era stato arbitro (nell'originale, infatti, vi è l'indicazione «Actum Soylii in hyp. arbitratoris D. Augustini de Salicibus» e nella traduzione si legge "Actum Soylii in casa et stuva del sudetto arbitrador Ser Augustino»), riguardo ad un tetto rotto con infiltrazioni d'acqua piovana, che le famiglie condividevano, precisando «ex originali quem legere potui bona fide et sine fraude transtuli in linguam vulgarem Italicam ${ }^{32}$.

Il fatto che un notaio, attivo a Soglio nel I569, abbia dovuto riprendere la controversia già trattata da Florio tre anni prima, traducendo un atto dal suo faldone, rappresenta un'ulteriore conferma che, per quella data, Michelangelo era già deceduto.

Il faldone degli atti notarili stilati a Soglio da Michelangelo in veste di notaio, oggi presso l'Archivio di Stato dei Grigioni, siglato B 663/2I (un tempo composto da 28 fogli, di cui rimangono solo gli ultimi I7), si chiude il 25 maggio del 1566 ed è lecito supporre che Michelangelo morì proprio nell'estate di quell'anno, con ogni probabilità a causa della tremenda epidemia di peste che nel 566 causò moltissimi vittime in Europa e giunse nei Grigioni in estate accompagnata da un'altra calamità: le piogge torrenziali (cfr. Rensch I9I4) ${ }^{33}$. Solo a Coira, dal luglio in avanti, morirono di peste I300 persone tra cui il Fabricius e Philipp Gallicius (con la moglie e tre dei suoi figli). Il cattolico Giambattista Crollalanza, nella Storia del contado di Chiavenna, non potendo fare a meno di sferrare attacchi contro i riformati, riferì:

Al primo sentore del fatal morbo, il ministro di Piuro, Girolamo Torriani, preso da spavento, era vilmente fuggito a Coira, mostrando ad evidenza come la vera carità non alligni nel cuore dei settari e sia esclusivo pregio di chi professa la vera fede! (Crollalanza I867: 2I8).

Crollalanza trasse l'informazione senza dubbio da una lettera malevola dell'anno precedente dello stesso Fabricius a Bullinger, datata 4 settembre I565:

Plurii quoque hoc malum ingravescit, unde minister illius ecclesiae Hieronymus ad nos profugerat. Displicuit mihi hoc in homine factum vehementer, quod is primus esset, qui fugam arriperet. Reheprendi eum. Respondit hunc morem esse ltalorum, templa statim claudi, omni congressu mutuo homines prohiberi. Sed quod eum alii quoque non pauci sequerentur, Senatus

32 «dall'originale che ho potuto leggere, in buona fede e senza frode, ho tradotto [l'atto] in lingua volgare italiana».

33 Si veda inoltre Jenny 1995: 22, che parlando della morte di Michelangelo afferma: «Vermutlich schon 157I, sicher am 29. Juni 1573 ist er samt seiner Frau tot, womöglich also der Pestepidemie von 1566 erlegen». Florio e la moglie risulterebbero dunque morti forse già nel I57I, certamente il 29 giugno I573, forse per l'epidemia di peste già nel 1566. 
noster eis hospitium negavit, jussit ad suos redire. Fortassis ad vos descendit, nusquam hic apparet amplius. Quod si se apud suos continuisset, consilio et re praesto fuisset, et saltem Baptismum administrasset. Certe videtur res pessimi exempli ideoque non ferendus sed ad suam Ecclesiam remittendus. Est alias homo perversi cerebri et judicii (Bullinger, Korrespondenz II).

Alla luce delle considerazioni di Fabricius, condivise ampiamente da Bullinger, che infatti nella sua risposta non mancò di menzionare il proprio esempio (disse che pur avendo perso la moglie e la figlia a causa dell'epidemia, non lesinava la propria presenza agli appestati), parrebbe che, al contrario di Torriano, Michelangelo Florio fosse pietosamente rimasto tra gli appestati della sua comunità, mettendo a repentaglio, come Bullinger e Fabricius, la propria vita e che, come quest'ultimo, avesse contratto il morbo.

La sua data di morte (e verosimilmente anche quella della moglie) va fissata dunque tra il giugno e il luglio della pestilenza del I566. Possiamo essere ancora più precisi a riguardo: sappiamo infatti, grazie a Die Liebestätigkeit der evangelischen Kirche Württembergs (Bossert 1905: I, 66-II7; 1906: I, 90), colossale lavoro d'archivio compiuto da Gustav Bossert agli inizi del Novecento, sino ad oggi mai consultato da chi si occupa della ricostruzione biografica di John Florio ${ }^{34}$, che John interruppe gli studi a Tübingen nel luglio del 1566 per rientrare in Bregaglia.

Non mi sembra lecito, sulla base dei documenti sin qui prodotti, affermare che vi sia alcun mistero attorno alla morte di Michelangelo Florio. Alla domanda di Panzieri «Ritornò in Inghilterra Michel Agnolo Florio o morì a Soglio?» (Panzieri 20I4: 42) la risposta mi pare chiara, così come è chiaro che chi non voglia considerare attendibili queste testimonianze, ipotizzando che Florio abbia trascorso ben quattro anni nascosto a Soglio (dove era noto a tutti e dove, come abbiamo visto, la sua abitazione era stata dismessa), per poi fuggire nel 1577 in Inghilterra, lo fa lavorando di fantasia.

Lo stesso Luigi Firpo aveva dedotto che Michelangelo Florio doveva essere morto nel I566, notando:

Quest'ultimo anno [1566] fu probabilmente l'ultimo della sua vita [...]. Si chiudeva così oscuramente un'esistenza torbida e burrascosa, non priva di vigoria intellettuale e di aggressivo dinamismo fra le tante della diaspora culturale italiana nella grande crisi civile e religiosa nel nostro Cinquecento (Firpo 1996: 259).

34 La mancata consultazione dell'opera di Bossert da parte di chi svolge ricerche su Florio forse è dovuta al fatto che la lettura del testo, ancora in caratteri gotici, non è particolarmente agevole per chi non sia a proprio agio con il tedesco. 


\section{Bibliografia}

Bainton, Roland H., Bernardino Ochino. Esule e Riformatore Senese del Cinquecento (I487-I563), Firenze, Sansoni, I940.

Bierbach, Mechtild, Travaux de linguistique et de philologie, 35-36, Paris, Klincksieck, I997.

Bonorand, Conradin, Reformatorische Emigration aus Italien in die Drei Bünde. Ihre Auswirkungen auf die kirchlichen Verhältnisse - Ein Literaturbericht, hrsg. vom Verein für Bündner Kulturforschung, Chur, Bündner Monatsblatt, 2000 (Beiheft 9 zum Bündner Monatsblatt).

Bossert, Gustav, «Die Liebestätigkeit der evangelischen Kirche Württembergs von der Zeit des Herzogs Christoph bis I650", Württembergische Jahrbücher, I905, I, pp. I-28; II, pp. 66-II7.

-. "Die Liebestätigkeit der evangelischen Kirche Württembergs von der Zeit des Herzogs Christoph bis I650», Württembergische Jahrbücher, 1906.

Bullinger, Heinrich, Korrespondenz mit den Graubündern, T. Schiess, Basel, Verlag der Basler Buch- und Antiquariatshandlung, I904-I906.

Bundi, Martin, Gewissensfreiheit und Inquisition im rätischen Alpenraum: demokratischer Staat und Gewissensfreiheit; von der Proklamation der "Religionsfreiheit» zu den Glaubens- und Hexenverfolgungen im Freistaat der Drei Bünde (I6. Jahrhundert), Bern, Haupt, 2003.

Busino, Giovanni, «Prime ricerche su Broccardo Borrone», Bibliothèque d'humanisme et renaissance, I962, 24, pp. I30-I67.

Camenisch, Emil, Storia della Riforma e Controriforma nelle valli meridionali del Cantone Grigioni e nelle regioni soggette ai Grigioni, Chiavenna, Valtellina e Bormio, Samedan, Engadin Press, 1950.

Cantimori, Delio, Eretici italiani del Cinquecento, Firenze, Sansoni, I939 (nuova edizione a cura di A. Prosperi, Torino, Einaudi, 2009).

Crivelli, Aldo, «Tessiner Handwerker und Künstler im Ausland», Schweizerische Bauzeitung, 1978, 96, pp. 866-875.

Crollalanza, Giovan Battista di, Storia del contado di Chiavenna, Milano, Serafino Muggiani, I867.

«Die Pfarrer der evangelischen Gemeinden in Graubünden und seinen ehemaligen Untertanenlanden", Jahresbericht der historisch-antiquarischen Gesellschaft von Graubünden, 1935, 65, pp. 95-298.

Du Cange, Charles du Fresne, sieur du, Glossarium medice et infimoe latinitatis, Niort, Favre, I883-I887.

Hoiningen-Huene, Christine von, «Mitteilungen aus Bergeller Notarsprotokollen, Protokollbücher Bergeller Notare aus der Zeit von I476 bis I594", Bündnerisches Monatsblatt: Zeitschrift für bündnerische Geschichte, Landes- und Volkskunde, I9I7, pp. 97-IO5, 20I-2II, 388-396; I9I9, pp. 57-6I, 85-95, I54-I60, I87-I89.

Firpo, Luigi, Scritti sulla Riforma in Italia, Napoli, Prismi, 1996. 
Giovanoli, Gaudenz, «Erinnerungen an hervorragende Pfarrer in Soglio», Bündnerisches Monatsblatt: Zeitschrift für bündnerische Geschichte, Landesund Volkskunde, 1932, 2, pp. 51-56.

Jenny, Beat Rudolf, «Die Übersetzungen von Agricolas "De re metallica" als Beispiel für die Verbreitung wissenschaftlicher Texte in den Landessprachen des I6. Jahrhunderts», Ferrum, I995, 67, pp. I6-25.

Kirk, Richard Edward Gent - Kirk, Ernest F., Returns of Aliens dwelling in the City and Suburbs of London from the Reign of Henry VIII to that of James I (HSQ X, I-5), Aberdeen, University Press, I900-I9Io.

Landeskarten der Drei Bünde, Chur, Calven, I974.

Martinoli, Gianna, Michel Angelo Florio: Un umanista "eretico" del Cinquecento tra Inghilterra e Grigioni, tesi sostenuta presso l'Università degli Studi di Milano, A.A. 1997/1998.

Memorie Domenicane, I948, 65-66, pp. I65-I66.

Panzieri, Corrado, La morte presunta di Michel Agnolo Florio, YoucanPrint Self-Publishing, 20I4.

Pellegrini, Giuliano, «Michelangelo Florio e le sue "Regole de la lingua thoscana"», Studi di filologia italiana, I954, 28, pp. 77-204.

Pool Georg, «Bergeller Notare», JHGG - Jahrbuch der Historischen Gesellschaft von Graubünden, 1983,II3, pp. 63-I54.

Rensch, Mathias, «La pestilenza (pechtialtyphus) el Grischun cunentginas autras notizias», Annalas da la Societad Retorumantscha, I9I4, 28, pp. 99I23.

Romani, Roberta - Bellini, Irene, Il segreto di Shakespeare. Chi ha scritto i suoi capolavori?, Milano, Mondadori, 2012.

Porta, Petrus Dominicus Rosius a, Historia Reformationis ecclesiarum raeticarum, II, Curiae Raetorum, Otto Verlag, I776.

Rossi, Carla, Italus ore, Anglus pectore. Studi su John Florio, I, London, TheCLA Academic Press, 20I8.

Rossi, Carla (a cura di), «La fede di battesimo di Michelangelo Florio, nato a Firenze, addì 28 settembre I5I 8 a hore I2», Theory and Criticism of Literature and Arts, 20I7, 2, I, pp. IO4-I20.

Roth Priska, Die Gemeinden im Bergell, Korporativ gedacht, genossenschaftich organisiert, feudal gehandelt (I4.-I6. Jahrhundert), tesi di dottorato sostenuta presso l'Universität Zürich, Aprile 2016.

[Salis], Familienarchiv von Salis. Regesten zu Pergamenturkunden, I. Serie bearbeitet von Pater Nicolaus von Salis-Soglio, Chur I898, dal 2012 online https:/www.gr.ch/DE/institutionen/verwaltung/ekud/afk/sag/dienstleistungen/bestaende/nichtstaatlichearchive/Documents/DVI_AI_SalisUrkunden_ISerie.pdf (consultato il I8.02.2019).

Strohm, Christoph, Ethik im frühen Calvinismus, Berlin, Gruyter, 1996. 
Truog, Jakob Rudolf, «Die Bündner Prädikanten I555-I90I nach den Matrikelbüchern der Synode», Jahresbericht der Historisch-Antiquarischen Gesellschaft von Graubünden, 1901, 3I, pp. 13-20.

Zagni, Luisa, Le pergamene del secolo XII della chiesa di S. Giorgio al Palazzo di Milano conservate presso l'Archivio di Stato di Milano (Pergamene milanesi dei secoli XII-XIII 5), Milano, Università degli Studi di Milano, 1988.

Zucchini, Giampaolo, «"In coercendis haereticis": l'esilio di Scipione Lentolo in Svizzera e il suo inedito epistolario (I567-I599)», in Studi politici in onore di Luigi Firpo, I, Milano, Franco Angeli, 1990, pp. 525-544.

\section{Indice dei manoscritti}

Bern

Burgerbibliothek

Bongarsiana Cod. A. 93. 7

Bongarsiana Cod. A. 93. II

Cambridge

University Library

Dd. XI. 46

Chur

Staatsarchiv Graubünden

Archivo di Soglio, Protocolli notarili, faldone XV

Cod. B 663/2I

Cod. B 663/27

Cod. D VI A I (Regesten zu Pergamenturkunden, I. Serie)

Cod. D VI SM / Cp I87

Cod. D VI SM / R 82 (Privatarchiv von Salis-St. Margrethen)

Cod. D VI So [2I/I24] II. A. 2, Quaderno IV

London

British Library

Sloane 30 II

Zürich

Staatsarchiv

E II 380 